\title{
USE OF A GEOGRAPHIC INFORMATION SYSTEM FOR DEFINING SPATIAL RISK FOR DENGUE TRANSMISSION IN BANGLADESH: ROLE FOR AEDES ALBOPICTUS IN AN URBAN OUTBREAK
}

\author{
MOHAMMAD ALI, YUKIKO WAGATSUMA, MICHAEL EMCH, AND ROBERT F. BREIMAN \\ Centre for Health and Population Research, International Centre for Diarrhoeal Disease Research, Bangladesh (ICDDR,B), \\ Mohakhali, Dhaka, Bangladesh; Department of Geography, Portland State University, Portland, Orgeon
}

\begin{abstract}
We used conventional and spatial analytical tools to characterize patterns of transmission during a community-wide outbreak of dengue fever and dengue hemorrhagic fever in Dhaka, Bangladesh in 2000. A comprehensive household-level mosquito vector survey and interview was conducted to obtain data on mosquito species and breeding as well as illness consistent with dengue. Clusters of dengue illnesses and high-density vector populations were observed in a distinct sector of the city. Dengue clusters are less identifiable in areas further away from major hospitals, suggesting that proximity to hospitals determines whether cases of dengue are diagnosed. Focusing on those areas relatively close to hospitals, we found a spatial association between dengue clusters and vector populations. Households reporting a recent dengue illness were more likely to have Aedes albopictus larvae present in the home when compared with households not reporting cases. Households reporting a recent dengue illness were also more likely to have a neighbor with Ae. albopictus present in the home. In contrast, the presence of Aedes aegypti within the premises as well as the homes of neighbors (within 50 meters) was not associated with dengue illness. Given that the breeding habitats for $A e$. albopictus are somewhat distinct from those of Ae. aegypti, the findings of this study have implications for control of dengue transmission in this urban setting where much of the focus has been on indoor mosquito breeding and transmission. Public health officials may find the disease-environment map useful for planning targeted interventions because it displays areas where transmission is most intense.
\end{abstract}

\section{INTRODUCTION}

An outbreak of dengue and dengue hemorrhagic fever occurred in Dhaka, Bangladesh in 2000. ${ }^{1}$ Dengue hemorrhagic fever had not been previously reported in Bangladesh. More than 5,000 hospitalized cases were identified between July 2000 and December 2000; there were more than 80 deaths (Ministry of Health, Bangladesh, Dengue Control Room, unpublished data).

Dengue is transmitted to humans, the only known reservoir of the disease, by Aedes mosquitoes. Adult female Aedes mosquitoes acquire the dengue virus by biting an infected human during the viremic phase, which usually lasts for 4-5 days, but may last up to 12 days. ${ }^{2,3}$ The virus is transmitted to other persons via bites from infected mosquitoes.

Aedes aegypti and Ae. albopictus have different breeding and biting patterns and these differences could influence potential for dengue transmission. These patterns could be relevant to efforts in progress to better define and control transmission of dengue viruses in Bangladesh. ${ }^{4-6}$ Efforts are also in progress to better define and control transmission of dengue viruses in Bangladesh.

The principle breeding sites of Aedes mosquitoes include containers such as cans, pots, earthen jars, plant pot bases, and drums, as well as tires and tree holes. ${ }^{7-9}$ Breeding sites of Aedes mosquitoes vary spatially. ${ }^{10}$ In a dengue control program, it is useful to identify precise areas of concentrated Aedes breeding. This information is particularly valuable if it can be linked with dengue incidence, since both the mosquito species and infected people are necessary for continued transmission. Areas with dense populations near breeding sites require interventions to minimize transmission.

Geographic information systems (GISs) are making practical contributions to public health. ${ }^{11}$ Identifying high-risk areas can be particularly useful for prioritizing prevention in public health decision-making. ${ }^{12-17}$ This study used a GIS to identify spatial risk for dengue. We measured spatial associa- tions between clusters of dengue cases and vector populations to identify areas of increased transmission. We produced a risk map so local health officials can target specific areas for focused interventions.

\section{MATERIALS AND METHODS}

Household survey. A household entomologic assessment, as well as an attitude and behavior survey, was conducted in Dhaka during the peak epidemic period for dengue/dengue hemorrhagic fever (August through October). Dhaka has an area of $144 \mathrm{~km}^{2}$ and an estimated population of 8.5-13.5 million people. ${ }^{18}$ The study was conducted within the Dhaka City Corporation area, which is administratively divided into 90 wards. The geographic area of each ward is referred to as a "polygon" in the GIS. The most recent population census, conducted in January 2001, showed that within the Dhaka City Corporation area the total number of households was $1,107,000$ and total population was $5,378,000 .{ }^{19}$ In the survey design method, a household was defined as one separate unit of accommodation (individual home or apartment) and the immediately surrounding premises, irrespective of the number of people residing within the unit. Approximately 100 houses (range $=100-119$ ) were selected randomly, based on frequency distribution of house types from each of the 90 wards. We collected information about distribution of housing types (i.e., independent, multistoried, semi-permanent, and slum house) from each of the ward commissioner's offices. We selected a proportional number of households in each ward according to the distribution of housing types within that ward (e.g., if in a specific ward $10 \%, 20 \%, 30 \%$, and $40 \%$ of the houses were of each respective housing type, we then selected 10, 20, 30, and 40 households, respectively, representing each type of house). A total of 9,462 houses with different housing structures were included in the entomologic survey. Permission to carry out this study was provided by the Ethical Review Committee of International Centre for Diar- 
rhoeal Disease Research, Bangladesh Centre for Health and Population Research. Signed informed consent was obtained from each household that participated in the study.

During the survey, the household head or other adult resident was asked whether anyone in the household had dengue/ dengue hemorrhagic fever diagnosed at health facilities during the past two months. We used these data to identify selfreported cases of dengue.

Field workers also looked for containers with standing water and for Aedes larvae within containers inside, outside, and on the roof of each household. A total of 44,532 larvae were collected, and were reared to their adult stage whenever possible for species identification. We used total vector populations (Ae. aegypti and Ae. albopictus) per household for the calculations to define clusters of vector populations.

We mapped each household using Magellan (San Dimas, CA) 315/320 global positioning system (GPS) receivers. An accuracy assessment of the receiver provided no differences in the reading obtained from Geoexplorer (Trimble Navigation, Ltd., Sunnyvale, CA) (a relatively expensive GPS receiver), which suggested that it was reasonable to use the less expensive receivers. Of the 9,462 houses, the GPS readings of 170 houses were found to be inaccurate (did not coincide within the Dhaka metropolitan area); thus, they were excluded while constructing the GIS. Two houses provided the same geographic coordinates in eight cases since they were observed to be very close to each other. A single point was referenced for each of those pairs; the household level data of the pairs were aggregated accordingly. Ultimately, we could construct the GIS with 9,284 reference points for houses. The spatial distribution of the houses surveyed under the entomologic assessment study is shown in Figure 1.

Defining spatial clusters. We used a raster GIS technology to analyze the spatially referenced data. The raster GIS divides space into a discrete number of cells called pixels. ${ }^{20}$ The geographic locations of the households are represented in pixels within the raster GIS. The spatial resolution of pixels was assigned 10 meters (typical distance between houses in many parts of Dhaka) to represent a house by a single pixel. By linking attribute identifications of the pixels with the entomologic data set, we implemented dengue cases and vector population in the raster GIS. Since the data type of dengue cases was binary and the data type of vector population was continuous, we used two methods of exploratory spatial data analysis: kernel estimation and kriging, respectively, to obtain smoothed data at a regular interval in space. The smoothed data were used to create surface maps from where the clusters of phenomena were isolated. The methods and procedures of the spatial data analysis and defining of the clusters are described in the next section.

Clusters of dengue cases. We used Kernel estimation to demonstrate areas of higher intensity of dengue cases. Each kernel takes account of observed data around a point of measurement, thus providing smooth estimates of univariate probability densities from a sample of observations. ${ }^{21-24}$ The mathematical expression for estimating kernel of the intensity of dengue cases at point $s$ is defined by

$$
\lambda(s)=\sum_{i=1}^{n} e_{i} w_{i}
$$

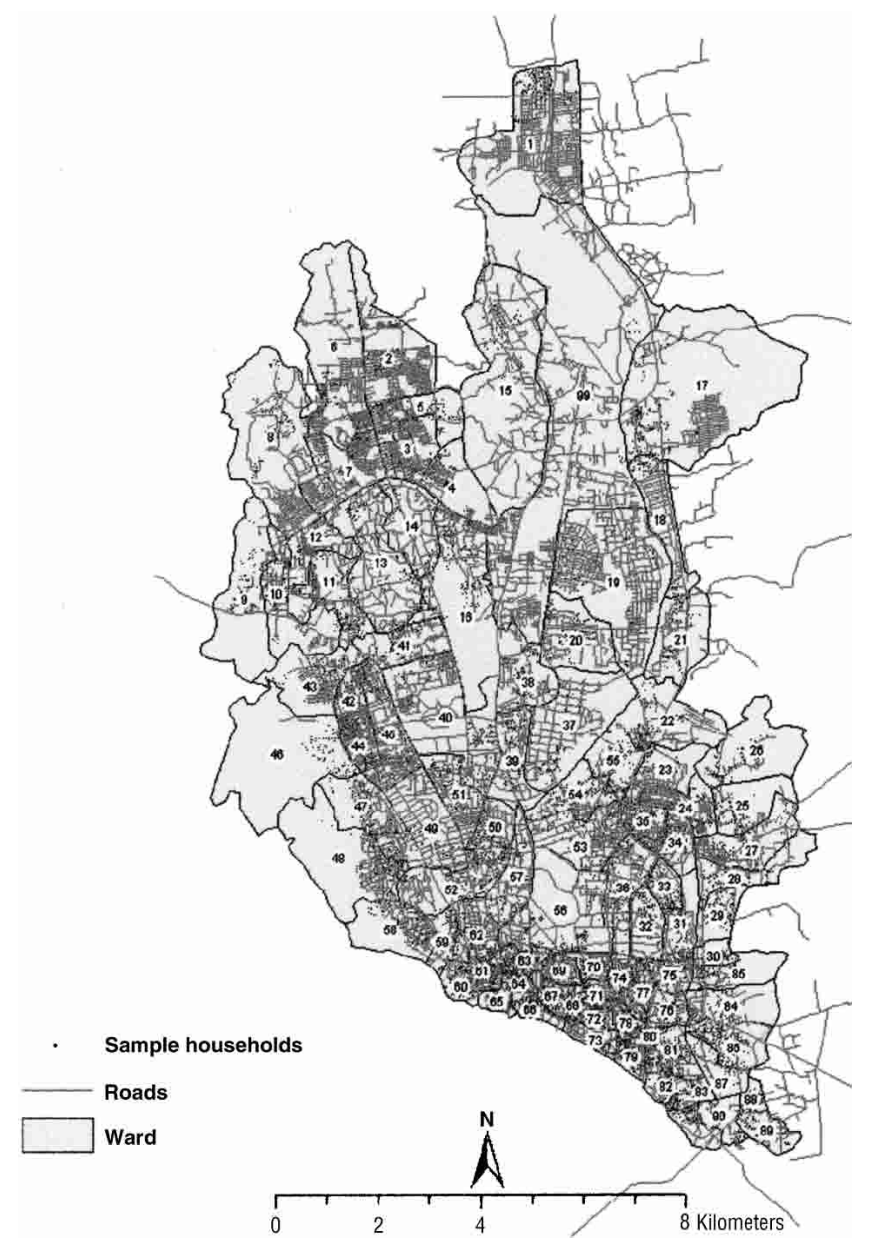

FIGURE 1. Spatial distribution of entomologic assessment survey households in Dhaka, Bangladesh. Ward 99 is not an administrative ward and was nor systematically evaluated in this study.

where $e_{i}, \ldots ., e_{n}$ are the observed cases at locations $s_{i}, \ldots, s_{n}$ respectively; $e_{i}$ has a value of 1 if a case is observed at $s_{i}$ and 0 otherwise. The further a point is from the point of measurement (kernel), the lesser the weight value (w) for computing the kernel. Thus, the kernel weight $\left(\mathrm{w}_{\mathrm{i}}\right)$ for the location $\mathrm{s}_{\mathrm{i}}$ is computed by

$$
w_{i}=\frac{\tau-d_{i}}{\tau}
$$

where $\tau$ is the maximum pixel distance ( 20 in this study [ $21 \times$ 21-pixel window]) and $d_{i}$ is the pixel lag between $s$ and $s_{i}$. We chose $21 \times 21$-pixel window representing a 44,100 $\mathrm{m}^{2}(210$ meters $\times 210$ meters) area. This provided an average of six data points for computing the intensity at each $s$, which yielded the best estimation for addressing local level (neighborhood-based) variation in the data. Note that in a $10,000 \mathrm{~m}^{2}$ (100 meters $\times 100$ meters) area, there would be only 2 data points, and 12 data points would have been provided if we covered a size of $90,000 \mathrm{~m}^{2}$ (300 meters $\times 300$ meters $)$ area. If we select a $10,000 \mathrm{~m}^{2}$ area, the data would display more individual variability, whereas a $90,000 \mathrm{~m}^{2}$ area would tend to blur local level variation in the data.

For 464 households, the respondents did not know whether dengue had occurred within the household; thus, 8,820 households were included in the analysis. The intensity of dengue 


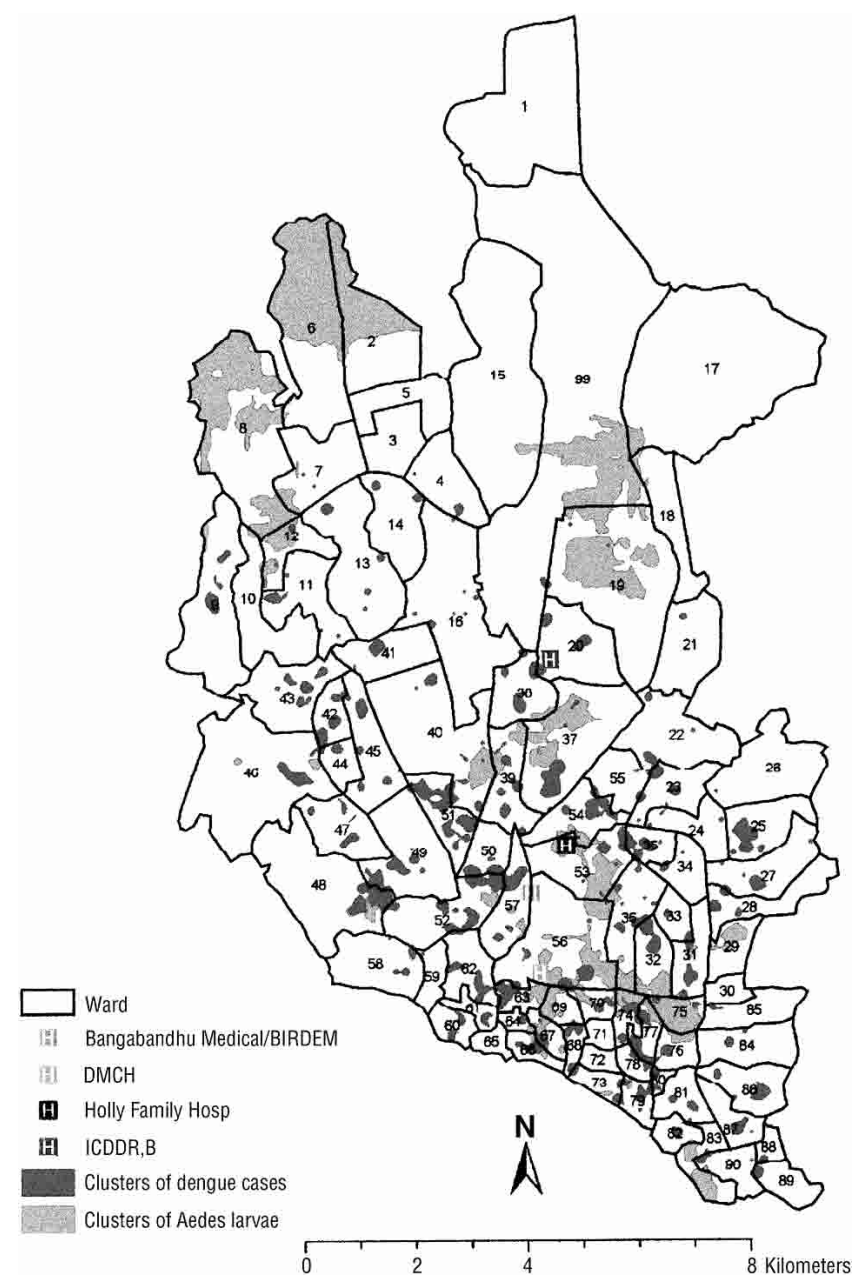

Figure 2. Clusters of dengue cases and vector populations in Dhaka, Bangladesh. BIRDEM = Bangladesh Institute of Research for Diabetics and Endocrinal Medicine; DMCH = Dhaka Medical College Hospital; Hosp = Hospital; ICDDR,B = International Centre for Diarrhoeal Disease Research, Bangladesh. This figure appears in color at www.ajtmh.org.

cases was then computed at a regular interval of 210 meters. Finally, using a contouring algorithm ${ }^{25}$ with the regularly spaced data, we created the surface of intensity of cases from where the clusters of higher intensity of cases were isolated. To isolate the clusters, we used an iterative process beginning with intensity, $\lambda(\mathrm{s})$, of 0.10 , increasing stepwise. At intensity of 0.58 , the iterative process yielded a visibly distinguishable threshold for defining higher intensity areas of dengue cases.

Clusters of vector populations. To identify clusters of higher density of vector populations, we began by interpolating the data on vector population using a geostatistical method called kriging, the estimates of which are unbiased and have minimum variance. ${ }^{26,27}$ The method is based on the assumption of spatial autocorrelation of data, and the autocorrelation structure (spatial distribution of the data) is addressed through variogram modeling. The underlying assumption of the variogram is that two observations close together are more similar than those further apart. Using the variogram in combination with observed data, we used ordinary kriging to interpolate the data at regular intervals (which yields intermediate locations, much like the way kernal values are calculated earlier in this report). An ordinary kriged estimate of a variable $\mathrm{Z}$ at a point $s$ is the weighted average of

$$
\gamma(s)=\sum_{i=1}^{n} \delta_{i} Z\left(s_{i}\right) .
$$

where $Z\left(s_{i}\right)$ is the observed data value at points $i$, and $\delta_{\mathrm{i}}$ is the weight associated with the data at point $i$, which is obtained from a variogram modeling.

Since the observed data on a vector population are highly skewed, the logged (natural) values were used instead in the variogram function and in kriging the data. A grid cell of size 210 meters was chosen to allow the data to be interpolated at a regular interval of 210 meters. Again, using a contour algorithm, we created the surface of density of the vector population, and the areas of higher density of the vector population were isolated from the surface map. Using a visual process to discriminate high and low vector density areas, we arrived at a cut-off value of 3.26 (logarithmic scale) to define high-density vector areas.

Spatial analysis. Spatial regression. Distance surfaces were created for each of the cluster maps to be used in a regression model for analyzing spatial association between the clusters of dengue cases and vector populations. In the distance surface, the further a pixel was from the cluster, the higher the attribute value was carried by the pixel. Distance surface was also created for the four major hospitals (Holy Family Red Crescent Hospital, Dhaka Medical College Hospital, Bangladesh Institute of Research for Diabetics and Endocrinal Medicine Hospital, and Bangabandhu Sheikh Mujib Hospital) where many dengue patients were admitted in 2000. The hospital distance surface was considered as the covariate in the multiple regression model.

The coefficient of determination $\left(\mathrm{r}^{2}\right)$ was computed to determine whether variances in clustering of dengue cases could be explained by independent factors (clusters of high density vector populations and proximity to the four major hospitals). In the regression models, the distance surface of dengue cases was entered as a dependent variable and the high density vector population as the independent variable.

\section{RESULTS}

A total of $872(9.4 \%)$ households were self-reported to have had at least one inhabitant with dengue. Aedes aegypti larvae were detected in 743 households $(8.0 \%)$, and Ae. albopictus larvae were present in 288 households (3.1\%). In addition, both species were detected in 281 households $(3.0 \%)$.

The spatial pattern map shows that self-reported dengue cases are clustered in the southern part of Dhaka in areas where the four major hospitals are located (Figure 2). The

TABLE 1

Simple spatial regression analysis results*

\begin{tabular}{lcc}
\hline \multicolumn{1}{c}{ Factor } & $\mathrm{r}$ & $\mathrm{r}^{2}$ \\
\hline Distance from the clusters of vector populations & 0.40 & 0.16 \\
Distance from the four major hospitals & 0.73 & 0.54 \\
Distance from the clusters of hospitalized cases & 0.73 & 0.54 \\
\hline
\end{tabular}

* The dependent variable is the distance from the cluster of high intensity of dengue cases. 


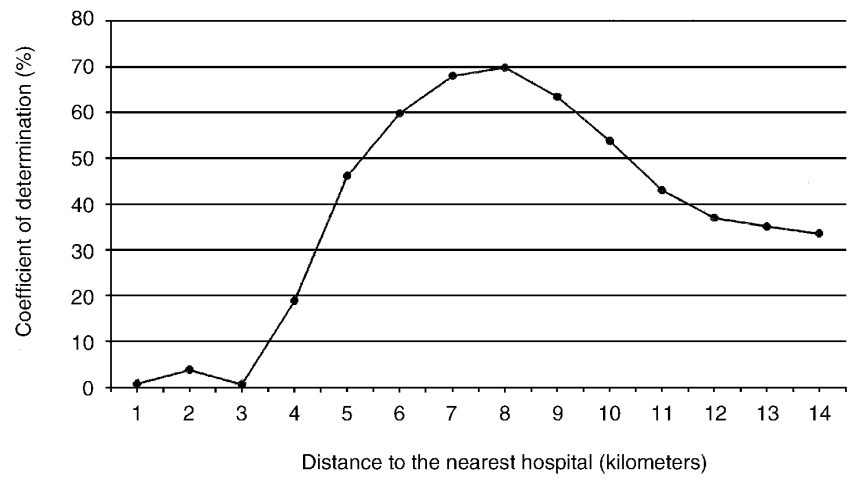

FIGURE 3. Spatial association between clusters of dengue cases and vector populations in Dhaka, Bangladesh by proximity to the major hospitals derived from analysis of progressively larger buffers.

results of the simple regression analysis are shown in Table 1. Approximately $54 \%$ of the variance $\left(r^{2}=0.54\right)$ in clustering of cases is explained by the distance to the hospitals; i.e., the greater the distance from the hospitals, the fewer the clusters of cases (Figure 3).

Within households where Ae. albopictus was found, $13.8 \%$ had at least one dengue case compared with $9.6 \%$ of the households where Ae. albopictus were not identified (relative risk $[\mathrm{RR}]=1.45,95 \%$ confidence interval $[\mathrm{CI}]=1.14-1.79$ ) (Table 2). In contrast, the presence of Ae. aegypti in the household premises was not associated with self-reported disease (Table 3). Using a GIS tool, we created a buffer of 50 meters around each of the households. Households within each buffer that reported a case of dengue and had Aedes larvae detected within the 50-meter buffer were counted. The neighborhood assessment was consistent with the household data in that the risk of acquiring dengue was increased when Ae. albopictus was present in and around neighbors' premises (within 50 meters of the home) $(\mathrm{RR}=1.24,95 \% \mathrm{CI}=$ 1.02-1.51) (Tables 4 and 5). The spatial pattern of households with Ae. albopictus and dengue cases is shown in Figure 4.

To understand the influence of population density over the clusters of cases, we superimposed the case cluster map on to a population density map (Figure 5). The mean population density was determined for the areas around each of the 190 case clusters. The median population density was $50,829 / \mathrm{km}^{2}$ for the case clusters compared with a median population density of $62,061 / \mathrm{km}^{2}$ for the 90 wards within Dhaka.

Clusters of vector population explain only $16 \%$ of the variance in clustering of cases (Table 1). When distance to the major hospitals was controlled using a multivariate model, approximately $56 \%$ of the variance in the clustering of dengue is explained by the clusters of vector population. Thus, the clustering of cases is linked to clustering of Aedes.

TABLE 2

Distribution of dengue cases and relative risk of disease by Aedes albopictus in household premises*

\begin{tabular}{lcrr}
\hline $\begin{array}{c}\text { Presence of Ae. albopictus } \\
\text { in the house }\end{array}$ & Case & \multicolumn{1}{c}{ No case } & \multicolumn{1}{c}{ Total } \\
\hline Yes (row \%) & $72(13.8)$ & $450(86.2)$ & $522(100)$ \\
No (row \%) & $800(9.6)$ & $7,498(90.4)$ & $8,298(100)$ \\
Total & 872 & 7,948 & 8,820 \\
\hline$*$ Relative risk $=1.45,95 \%$ confidence interval $=$ & $1.14-1.79$.
\end{tabular}

TABLE 3

Distribution of dengue cases and relative risk of disease by Aedes aegypti in household premises*

\begin{tabular}{lllr}
\hline $\begin{array}{l}\text { Presence of Ae. aegypti } \\
\text { in the house }\end{array}$ & \multicolumn{1}{c}{ Case } & \multicolumn{1}{c}{ No case } & \multicolumn{1}{c}{ Total } \\
\hline Yes (row \%) & $102(10.9)$ & $837(89.1)$ & $939(100)$ \\
No (row \%) & $770(9.8)$ & $7,111(90.2)$ & $7,881(100)$ \\
Total & 872 & 7,948 & 8,820 \\
\hline * Relative risk $=1.11,95 \%$ confidence interval $=0.91-1.35$.
\end{tabular}

The influence of hospital distance on spatial association between clusters of dengue and vector population was further investigated by analyzing spatial subsets. Fourteen buffer zones starting from 1 to $14 \mathrm{~km}$ of radius from the center of the four major hospitals were created. Separate regressions were modeled with the data of the fourteen spatial subsets. The first data set included a $1-\mathrm{km}^{2}$ area around the four hospitals and the last included a $14-\mathrm{km}^{2}$ area around the hospitals. Eventually, there were more data values for the larger buffer zones. No significant spatial association was observed between the clusters when the analysis was restricted to a small ( $<5 \mathrm{~km}$ radius) or a large buffer zone ( $>11 \mathrm{~km}$ radius) (Figure 3 ). The buffer zone of $8-\mathrm{km}$ radius shows the highest degree of spatial association (coefficient of determination $=$ 0.70 ) between the clusters.

\section{DISCUSSION}

This study suggests that the presence of Ae. albopictus in or around household premises was associated with an increased risk of dengue transmission. Although Ae. albopictus larvae were present in a lower number of households than $A e$. aegypti, they seemed to be linked with transmission. Studies of dengue transmission have suggested that Ae. albopictus are more likely than Ae. aegypti to breed and transmit disease outside of the home ${ }^{4,5}$ and within rural or semi-urban areas. ${ }^{6}$ Conversely, Ae. aegypti are traditionally felt to be the principal vector in urban areas and during epidemics. ${ }^{28,29}$ Aedes albopictus breeds in tree holes and cut bamboo, as well as within tin cans, used tires, and flower pots. ${ }^{30}$ Although the associations we observed were statistically significant they were not huge; however, the trends may be important given that the findings are limited by the timing of our investigation (weeks or months after the illness) and the restrictions on our ability to look for and find all potential breeding sites within 50 meters of the home. Identifying a key role for Ae. albopictus in transmission presents additional public health considerations for control of epidemic dengue in this densely populated urban area, where thus far interventions have focused primarily on Ae. aegypti.

\section{TABLE 4}

Distribution of dengue cases and relative risk of disease by Aedes albopictus within 50 meters of households*

\begin{tabular}{lcrr}
\hline $\begin{array}{c}\text { Presence of Ae. albopictus } \\
\text { in the house }\end{array}$ & \multicolumn{1}{c}{ Case } & \multicolumn{1}{c}{ No case } & \multicolumn{1}{c}{ Total } \\
\hline Yes (row \%) & $92(10.2)$ & $809(89.8)$ & $901(100)$ \\
No (row \%) & $1,479(8.2)$ & $16,492(91.8)$ & $17,971(100)$ \\
Total & 1,571 & 17,301 & 18,872 \\
\hline$*$ Relative risk $=1.24,95 \%$ confidence interval $=1.02-1.51$. &
\end{tabular}


TABLE 5

Distribution of dengue cases and relative risk of disease by Aedes aegypti within 50 meters of households*

\begin{tabular}{lccr}
\hline $\begin{array}{c}\text { Presence of Ae. aegypti in } \\
\text { the house }\end{array}$ & \multicolumn{1}{c}{ Case } & \multicolumn{1}{c}{ No case } & \multicolumn{1}{c}{ Total } \\
\hline Yes (row \%) & $184(8.6)$ & $1,960(91.4)$ & $2,144(100)$ \\
No (row \%) & $1,387(8.3)$ & $15,341(91.7)$ & $16,728(100)$ \\
Total & 1,571 & 17,301 & 18,872 \\
\hline
\end{tabular}

* Relative risk $=1.04,95 \%$ confidence interval $=0.88-1.22$.

This study identified clusters of dengue cases and high vector populations. Areas where the clusters of dengue cases and high vector populations either intersect or are close to each other may be subject to further high transmission of dengue, since both infected persons and vector populations are needed for transmission of the disease. Thus, our observation that population density did not independently correlate with incidence of disease is not surprising, since presence of an infected vector population is the critical factor.

Dengue clusters were also less frequently observed in areas far from the four major hospitals, often despite the presence of dense vector populations. Dengue cases that had occurred in those areas may not have been diagnosed or recognized because many people who live further away may not reach a hospital, which is the setting where dengue diagnosis is most often made in Dhaka. This may have weakened the association we observed between the clusters of dengue cases and vector populations. The poor spatial association when considering larger distances $(>11 \mathrm{~km})$ from hospitals suggests that hospital-based surveillance for dengue would substantially underestimate the true disease burden.

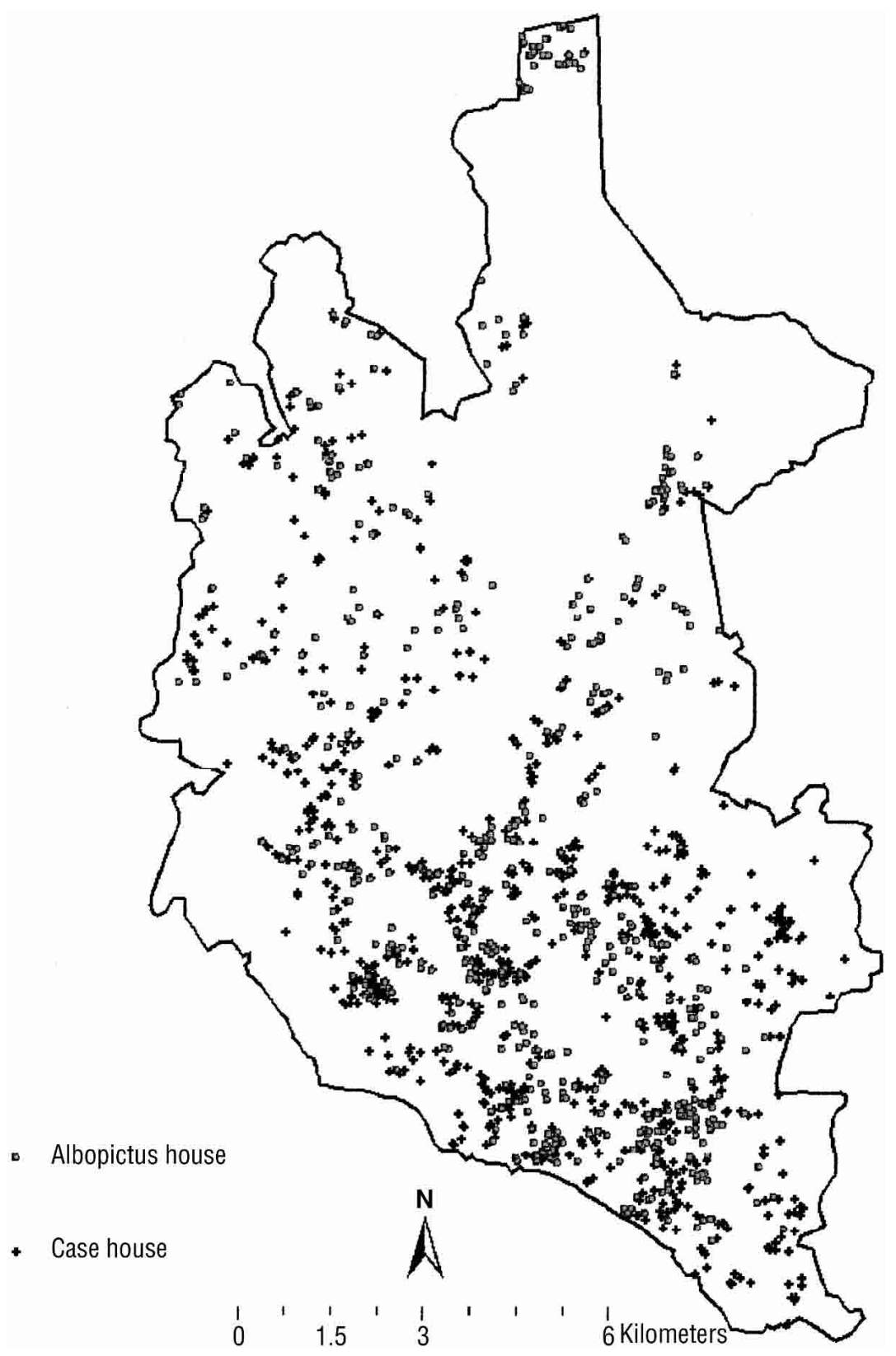

FIGURE 4. Spatial distribution of cases and houses in Dhaka, Bangladesh where Aedes albopictus were identified. 


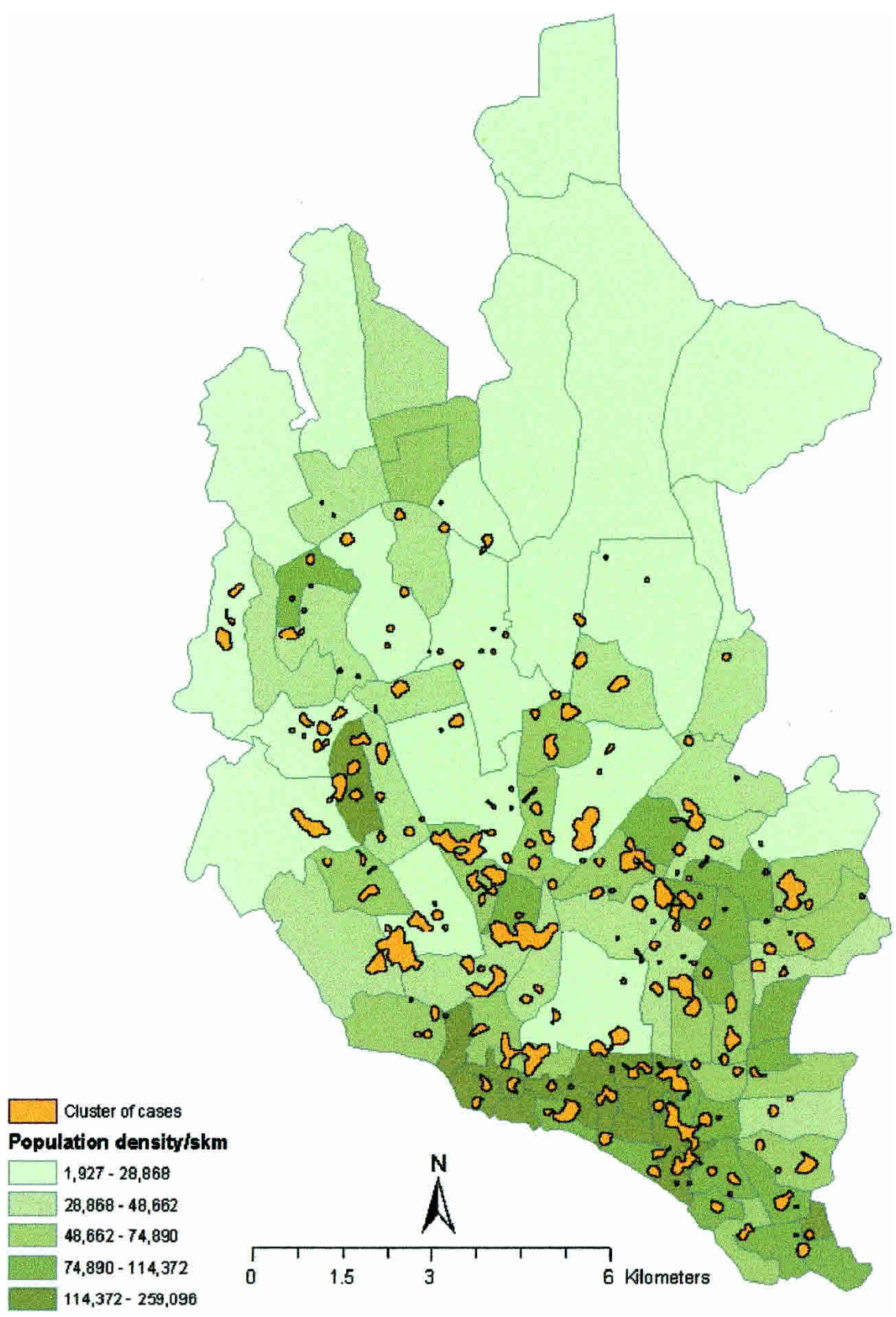

FIGURE 5. Clusters of dengue cases in Dhaka, Bangladesh superimposed on a map of the ward population density (quantile distribution). $\mathrm{skm}=$ square kilometers. This figure appears in color at www.ajtmh.org.

Weak spatial associations between dengue clusters and vector populations at very short distances from hospitals may be attributed to the characteristics of these areas. The hospitals are located in commercial areas where relatively fewer people live. Since dengue infection appears to be a recent phenomenon in Bangladesh, it is possible that the proportion of Aedes mosquitoes that are infected with dengue virus is not homogenous around the city; thus, the relationship between vector density and disease incidence might not yet be consistent.

This study was conducted early in the dengue outbreak before geographic information on laboratory-confirmed cases was routinely collected during surveillance. We relied on selfreporting of cases, which limits the precision of our findings since quality of diagnosis may vary by health facilities and patient recall and/or comprehension may not always be optimal. Self-reporting may result in pseudo-clustering because residents living near a true case of dengue may have been more aware of the disease and might have been more likely to assume that they also had dengue fever.

Mayer ${ }^{31}$ suggested that "the human-environment relationship, if disturbed enough by major changes in land use, migration, population pressure, or other stressors can show significant maladaptation, as manifested by the appearance or diffusion of new diseases." Thus, the clustering of vector populations in distinct sectors of the city suggests that there might have been some behavioral or environmental processes operating at neighborhood levels that supported breeding of Aedes mosquitoes and/or transmission of dengue viruses. 
Studies are underway to define factors responsible for clustering of vectors. Multi-temporal satellite imagery can be used to model the local level environmental characteristics (such as vegetation type, bodies of water, densely populated communities) that might promote breeding of disease-transmitting vectors. $^{32}$

Until a safe and effective vaccine is available, controlling Aedes mosquitoes and improved case management are the principal options available for reducing the burden of dengue in Dhaka. An integrated control measure ${ }^{33}$ targeting highrisk areas is a logical approach to minimize the impact of disease, given the limited resources of this country. This paper identifies a strategy of using spatial risk analysis with human disease and vector surveillance data for targeting areas for integrated interventions to reduce burden of disease during outbreaks of dengue and dengue hemorrhagic fever.

Received December 2, 2002. Accepted for publication July 23, 2003.

Acknowledgments: We are grateful for the cooperation and collaboration of the scientists at International Centre for Diarrhoeal Disease Research, Bangladesh (ICDDR,B). We are also grateful to Touhid Uddin Ahmed, Professor M. Ismail Hossain, and Dr. Manjur A. Chowdhury for their technical assistance on entomologic data collection. We thank Zahirul Huq and Mamunur Rashid for their assistance in constructing the GIS database.

Financial support: This research was funded by the United States Agency for International Development, Duncan Brothers, Ltd., the American Express Foundation, the Department for International Development of the United Kingdom, and the ICDDR,B: Centre for Health and Population Research, which is supported by the countries and agencies that share its concern for the health problems of developing countries. Current donors providing unrestricted support include the aid agencies of the Governments of Australia, Bangladesh, Belgium, Canada, Japan, the Netherlands, Sweden, Sri Lanka, Switzerland, the United Kingdom, and the United States of America; international organizations include United Nations Children's Fund.

Authors' addresses: Mohammad Ali, International Vaccine Institute, Seoul National University Campus, Shillim-dong Kwanak-ku, Seoul, Republic of Korea 151-742, Fax: 82-2-872-2803, E-mail: mali@ivi.int. Yukiko Wagatsuma and Robert F. Breiman, Centre for Health and Population Research, International Centre for Diarrhoeal Disease Research, Bangladesh (ICDDR,B), Mohakhali, Dhaka, Bangladesh, E-mail: breiman@icddrb.org. Michael Emch, Department of Geography, Portland State University, Portland, OR 97207-0751.

\section{REFERENCES}

1. Rahman M, Rahman K, Siddique AK, Shoma S, Kamal AHM, Ali KS, Nisaluk A, Breiman RF, 2002. First outbreak of dengue hemorrhagic fever, Bangladesh. Emerg Infect Dis 8: 738740.

2. Gubler EJ, Suharyano W, Tan R, Abidin M, Sie A, 1981. Viraemia in patients with naturally acquired dengue infection. Bull World Health Organ 59: 623-630.

3. Gubler DJ, 1988. Dengue. Monath TP, ed. The Arboviruses: Epidemiology and Ecology. Boca Raton, FL: CRC Press, 223-260.

4. Ishak H, Miyagi I, Toma T, Kamimura K, 1997. Breeding habitats of Aedes aegypti (L) and Aedes albopictus (Skuse) in villages of Barru, South Sulawesi, Indonesia. Southeast Asian J Trop Med Public Health 28: 844-850.

5. Yadav RS, Sharma VP, Chand SK, 1997. Mosquito breeding and resting in treeholes in a forest ecosystem in Orissa. Indian $J$ Malariol 34: 8-16.

6. Knudsen AB, 1995. Global distribution and continuing spread of Aedes albopictus. Parassitologia 37: 91-97.

7. Nasiruddin M, 1952. Mosquitoes breeding in tree holes and bamboo stumps in Dacca (East Pakistan). Pakistan J Health 2: 110-112.

8. Khan AR, 1980. Studies on the breeding habits and seasonal prevalence of larval population of Aedes aegypti (L.) and Aedes albopictus (Skuse) in Dacca City. Bangladesh Med Res Bull 6: 48.

9. Ahmed TU, Joshi GP, Ahmed RU, 1990. Container habitat mosquitoes of Bangladesh. $J$ Zool 5: 7-16.

10. Su MD, Chang NT, 1994. Framework for application of geographic information system to the monitoring of dengue vectors. Kao Hsiung I Hsueh Ko Hsueh Tsa Chih 10: S94-S101.

11. Jacquez GM, 2000. Spatial science in epidemiology: nascent science or a failure of GIS. J Geograph Syst 2: 91-97.

12. Cliff AD, Haggett $P, 1996$. The impact of GIS on epidemiological mapping and modelling. Longley P, Batty M, eds. Spatial Analysis: Modelling in a GIS Environment. Stoke on Trent, United Kingdom: Pearson Professional, Ltd., 321-343.

13. Mason TJ, 1995. The development of the series of U.S. cancer atlases: implications for future epidemiologic research. Stat Med 14: 473-479.

14. White AA, 1995. Mapping and geographic display of data. Stat Med 14: 697-699.

15. White AA, Croner CM, 1992. Geographic Information Systems (GIS) Activity in the U.S. Department of Health and Human Services. Alexandria, VA: American Statistical Association. Proceedings of the Government Statistics Section, 84-89.

16. Croner CM, Pickle LW, Wolf DR, White AA, 1992. A GIS approach to hypothesis generation in epidemiology. Technical papers, ASPRS/ACSM/RT92. Proc GIS Cartography 3: 275283.

17. Bertollini R, Martuzzi M, 1999. Disease mapping and public health decision-making: report of a WHO meeting. Am J Public Health 189: 780.

18. Afsar R, 2000. Rural-Urban Migration in Bangladesh: Causes, Consequences and Challenges. Dhaka, Bangladesh: The University Press, Limited.

19. Bangladesh Bureau of Statistics, 2002. Household and Population Data of Statistical Metropolitan Areas, 2001 (enumerated). Statistical Pocketbook. Dhaka, Bangladesh: Statistics Division, Ministry of Planning. Government of the People's Republic of Bangladesh.

20. Eastman JR, 1997. Idrisi for Windows: User's Guide Version 2.0. Worcester, MA: Clark University.

21. Robinson GM, 1998. Methods and Techniques in Human Geography. New York: John Wiley \& Sons.

22. Gatrell AC, Bailey TC, Diggle PJ, Rowlingson BS, 1996. Spatial point pattern and its application in geographical epidemiology. Trans Inst Br Geogr 21: 256-274.

23. Silverman D, 1986. Density Estimation for Statistics and Data Analysis. London: Chapman and Hall.

24. Bailey TC, Gatrell AC, 1995. Interactive Spatial Data Analysis. London: Longman Scientific \& Technical.

25. Surfer, 1999. Surfer ${ }^{\circledR} 7$ User's Guide: Contouring and $3 D$ Surface Mapping for Scientists and Engineers. Golden, CO: Golden Software, Inc.

26. Oliver MA, Webster R, 1999. Kriging: a method of interpolation for geographical information systems. Int J Geogr Information Systems 4: 313-332.

27. Webster R, Oliver MA, Muir KR, Mann JR, 1994. Kriging the local risk of rare disease from a register diagnoses. Geogr Anal 26: $168-185$.

28. Chung YK, Pang FY, 2002. Dengue virus infection rate in field populations of female Aedes aegypti and Aedes albopictus in Singapore. Trop Med Int Health 7: 322-330.

29. Choochote W, Tippawangkosol P, Jitpakdi A, Sukontason KL, Pitasawat B, Sukontason K, Jariyapan N, 2001. Polygamy: the possibly significant behavior of Aedes aegypti and Aedes albopictus in relation to the efficient transmission of dengue virus. Southeast Asian J Trop Med Public Health 32: 745-748.

30. Hawley WA, 1988. The biology of Aedes albopictus. J Am Mosq Control Assoc (Suppl 1): 1-39.

31. Mayer JD, 2000. Geography, ecology and emerging infectious disease. Soc Sci Med 50: 937-952.

32. Hay SI, 1997. Remote sensing and disease control: past, present and future. Trans R Soc Trop Med Hyg 91: 105-106.

33. Wang $\mathrm{CH}$, Chang NT, Wu HH, Ho CM, 2000. Integrated control of the dengue vector Aedes aegypti in Liu-Chiu village, PingTung County, Taiwan. J Am Mosq Control Assoc 16: 93-99. 